\title{
Lighting solutions of sales area according to the preferences of the target groups
}

\begin{abstract}
In some stores you feel better than others and it's not always a question of exposed assortment, or arrangement of space. There's "something" that we often do not realize it's the lighting. Light effects mood and emotions - both positive and negative direction. The question still remains, which parameters have lighting in retail environments greatest efficiency and lighting options that have a positive effect on the emotional perception. These situations and whether they have different target groups, individual preferences with regard to light are filling a large study conducted by Zumtobel Lighting along with Numphenburg Consult Gruppe AG. In this study used methods from the field of neuroscience and psychophysiology, and through them, some of the above questions answered. Found, for example, that the lighting parameters most affect human emotions and composition parameters which has a positive effect on the mood of customers. In addition, using a neuropsychological model of limbic types (Limbic ${ }^{\circledR}$ Type) analyzed preference lighting at seven different target groups.
\end{abstract}

Keywords: Limbic® Lighting; lighting solutions of sales areas

\section{Sumary}

Light impacts on mood and emotions - both in positive and negative ways. The question so far is, which lighting parameters, in a retail environment, possess the greatest effect, and which lighting scenarios have a positive impact on emotional perception. Neither these situations nor the question if different target groups have individual preferences with respect to light have ever been investigated yet.

Using methods from neuroscience and psychophysiology, several of these questions have now been answered in the course of this study. For instance, valuable clues were found as to which lighting parameters most strongly affect people's emotions and which composition of lighting parameters will influence the customers' mood most positively. Additionally, the lighting preferences of seven different target groups were analysed using the neuropsychological target group model Limbic $\AA$ Type.

The results show that there are clear differences with respect to lighting preferences between the individual target groups. In turn, accordingly, there is no single scenario that triggers the same positive emotions in all target groups. Rather, each target group seems to have its own requirements regarding light. The target group-specific results of this study have allowed sectioning into three large target group clusters, with target group preferences being similar within each individual cluster.

In developing lighting concepts for the retail sphere, the findings obtained may help to increase customers' wellbeing during general shopping, and accordingly also the amount of time they spend in a store.

\section{Background}

Over the past two decades, neuroscientists have been able prove that emotions are the main drivers of the way we act and behave. Only people, situations and products that trigger our emotions are of any importance to the brain. Thus, it is only our emotions that infuse our everyday environment with meaning.

It is now a known fact that more than 95 per cent of human decisions are made unconsciously. Purchasing decisions are also made on the basis of emotional processes, and not by our intellect, as had been assumed so far. Brain science, accordingly, has dropped the concept of rational and conscious homo sapiens long ago.

These findings suggest that classic tools of market research, based on explicit surveys, should be questioned. Verbally requested assessments do not always coincide with what customers feel on an emotional level and with how they ultimately behave.

For some time now, we have come to consider the customer as an "emotional consumer". This is where neuromarketing comes in, taking advantage of recent findings from brain research, as well as, from equipmentbased, neuroscientific methods in order to decode unconscious decision-making processes and emotional events.

This "neuroscientific" approach addresses numerous issues. For instance, brands, products, retail outlets, and other topics have been examined using the methods from neuromarketing.

Light is another topic that is highly relevant in the context of the marketing of products, especially in the area of shop\&retail; it is a well established fact that light is able to influence the mood of people in a positive, as well as, negative way, and that it may accordingly have an impact on (purchasing) behaviour. However, formerly there had not been any investigation into precisely which lighting parameters have which influence on human emotions.

In this complex study, the type of emotional reactions that people will display to various lighting scenarios has now been investigated. Specifically, the investigators looked into the question of what kind of interaction between different lighting parameters will trigger the most positive emotions in customers and whether there is any, one lighting scenario that affects all people equally positively, or if different target groups have very special lighting preferences.

For this purpose, a novel method developed by Gruppe Nymphen burg Consult AG was applied: Limbic ${ }^{\circledR}$ Emotional Assessment, or LEA in brief.

\section{Method}

Limbic $\AA$ Emotional Assessment is based on the methods of neuroscience and psychophysiology. The latter discipline deals with the connections between emotional 
processes in the brain and the concomitant physical reactions.

Due to the combination with Limbic $\AA$, a tried and tested brand and target group navigation model (see graphic 1), LEA allows for differentiation according to specific target groups.

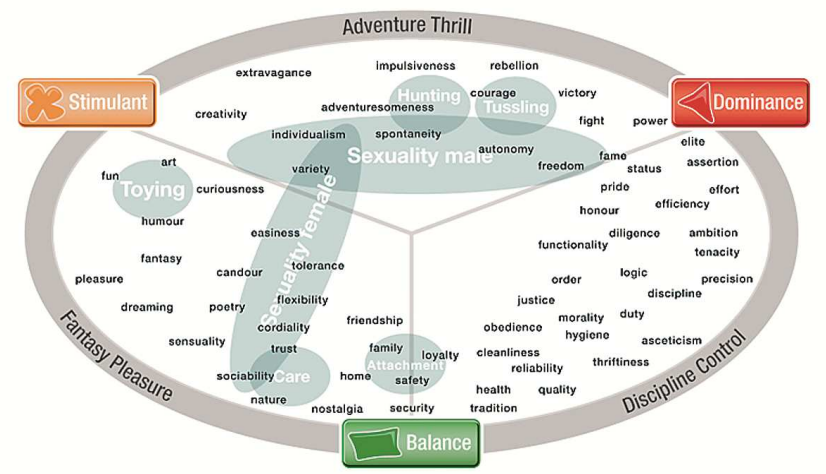

Graphic 1: Limbic® Map: Three emotional systems and the motive sectors of humans () Gruppe Nymphenburg

One brief example to explain the method: just imagine you are lying in bed at night and witness a burglar entering your house or flat.

In such situations, physical and cognitive reactions are measurable, such as strong heart palpitations, hot flashes, and a sense of extreme alertness. These reactions suggest the emotional perception, the level of activation and awareness of a person, as well as behavioural tendencies. In case of a burglary it would be obvious very quickly that the person feels anxiety and extreme agitation, that he/she shows a high level of alertness, as well as the urge to get out of this situation as quickly as possible.

Not all emotions are as obvious as those in the above example. Therefore, the scientifically tested LEA method combines five different parameters that are able to record even the smallest physical reactions.

From brain waves via skin conductance up to cardiovascular (heart) activity, numerous physical responses of customers are measured to draw conclusions about the emotional state of the person concerned.

For the present study, the LEA served the purpose of understanding target group specific lighting preferences even better and help provide some insights into the development of target group specific lighting solutions and lighting technologies.

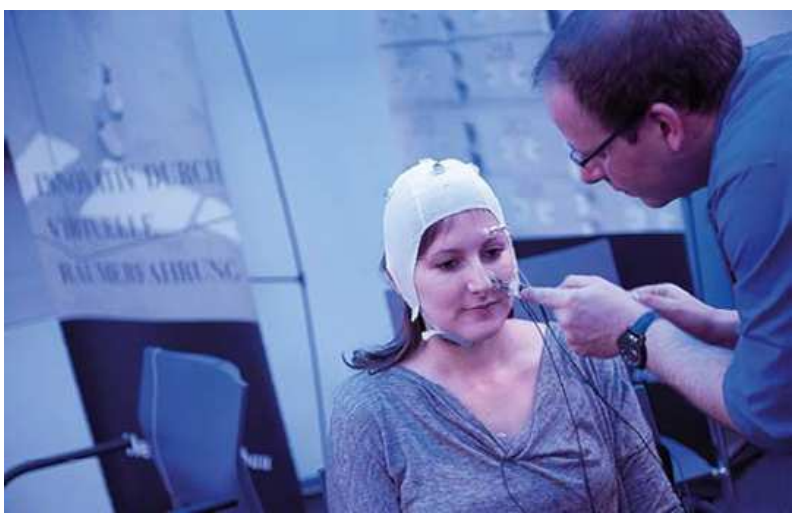

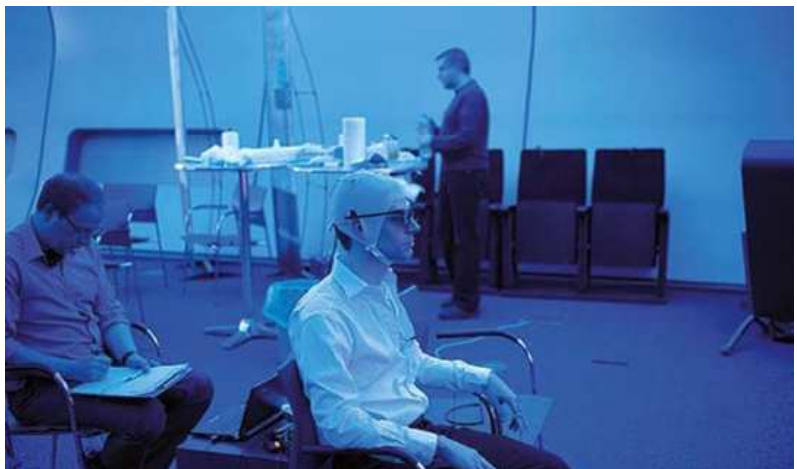

Fig. $1+2$ : Utilization of LEA

\section{Study details}

In order to model the reality of shops or retailers as accurately as possible, a 3D simulation of a fashion store was installed at Terminal V in Lauterach (Austria) in January 2013 (see Fig. 3).

This complex installation, conveyed to the test subjects the illusion of actually being at the POS. At the same time, a standardised laboratory setting was created to ensure valid comparisons of the reactions of all participants.

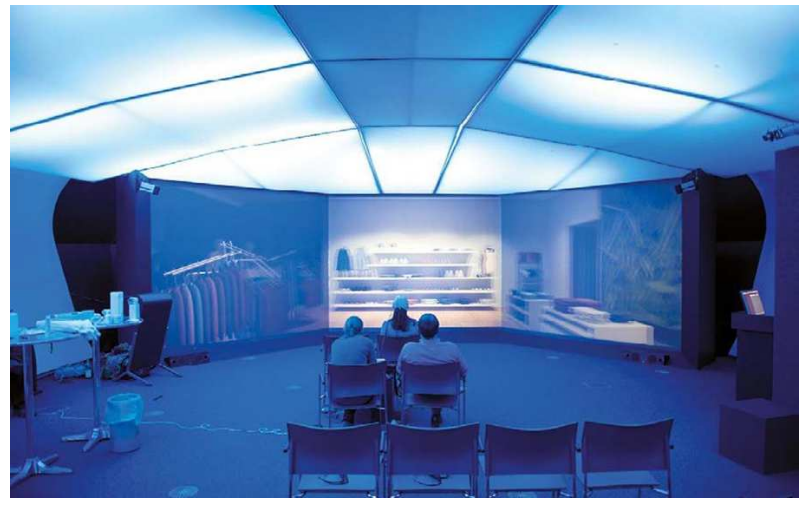

Fig. 3: 3D visualisation of a fashion store at Terminal V in Lauterach, Austria

Within this 3D visualisation, a total of 20 different lighting scenarios featuring various combinations of 6 lighting parameters were tested.

The parameters were the following:

- Light colour

- Light output

- Light distribution diffuse/directional

- Light distribution/beam pattern

- Contrasts/brightness

- Contrasts/light colour

Overall, three lighting scenarios per parameter $1-5$ and five lighting scenarios for parameter 6 were developed (Fig. 4). 


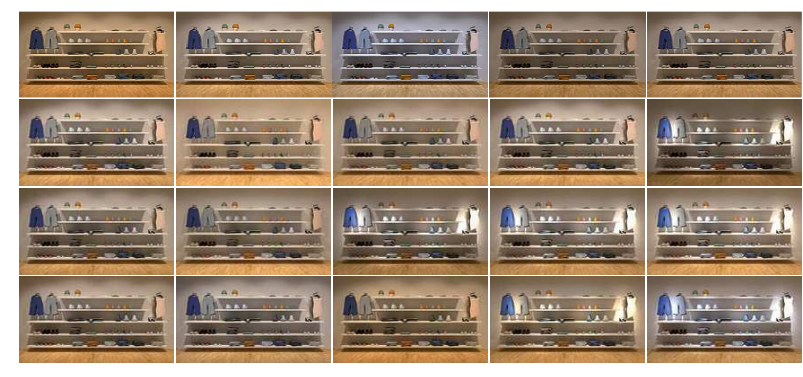

Fig. 4: 3D illustration of the lighting scenarios tested

\section{a. Participants}

For this study, 48 test subjects ( 25 women and 23 men between 19 and 62 years of age) were recruited.

Before the test, the participants were categorised and selected according to different target groups, the socalled Limbic $\AA$ Types (see Graphic 2), using a questionnaire.

The Limbic $\AA$ Types form a segmentation within the Limbic $\circledast$ model and focus on the complex emotional personality structures of consumers. Socio demographic details such as age, sex and income are negligible in this context.

The Limbic $\AA$ Types enable us to obtain a great variety of analysis across all areas of consumer and media usage behaviour.

There are great differences among consumers in terms of purchasing and consumer behaviour. The differences between their individual personalities are of utmost importance for consumer behaviour and they are primarily due to different characteristics of the system of emotions and motivations in the brain.

According to the Limbic $\AA$ model, there are essentially three major emotional systems in human beings: the balance, the stimulance and the dominance system. Beyond that, there are also mixed forms such as imagination/pleasure, adventure/thrill, as well as discipline/control.

These individual emotional focuses, influence the customer's purchasing behaviour and buying decisions mostly on an unconscious level.

A total of seven limbic types were derived from the above-mentioned motivational and emotional spheres; they are the following:

- Disciplinarians

- Traditionalists

- Harmonisers

- Open-minded

- Hedonists

- Adventurers

- Performers

All seven Limbic $\AA$ Types were represented in the study described herein by at least 6 participants per type.

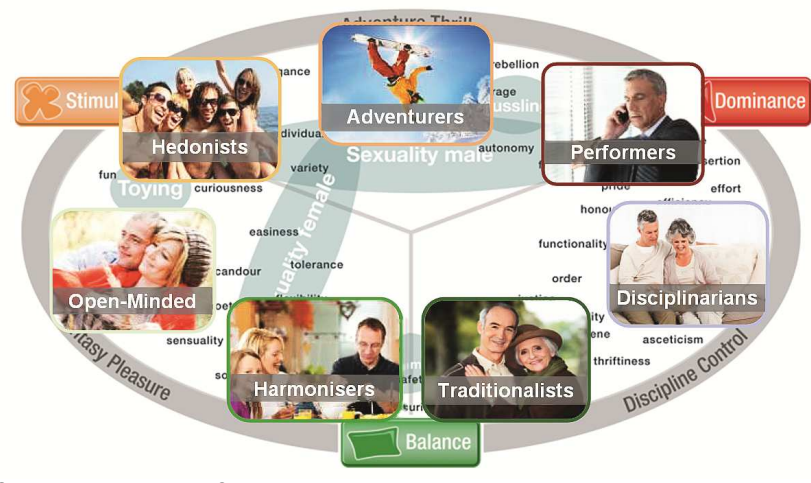

Graphic 2: The 7 Limbic $\circledast$ Types

\section{b. Realisation}

On the day of the study, the test subjects were welcomed by the investigator one after the other; they were informed about the study and given instructions. Electrodes were then placed on the participants' heads, fingers, on the insides of their wrists, their chests and faces, after which they were installed in front of the 3D installation. To enable them to see the 3D effect, the test subjects were also given 3D spectacles.

Subsequently, a one minute baseline measurement was carried out to record the participants' physical reactions at rest, and to allow for meaningful comparisons between test subjects.

After that, the participants were shown the various lighting scenarios (20 in total) in randomised sequence, alternating in each case with a control scenario. Each lighting scenario was displayed for a period of 20 seconds. In order to watch the lighting scenarios undisturbed, the participants were not given any other tasks during data collection.

Finally, the wiring was removed from the test subjects, after which they were seen off by the investigator.

A complete trial took 45 minutes.

\section{Data analysis}

The data was evaluated by means of a complex discriminant analysis. In concrete terms, this means that the changes of all participants' physical responses to the lighting scenarios tested were processed in a joint multivariable statistical analysis.

Thus, an emotional "response range" was defined where the locations of the test subjects' emotional reactions to each lighting scenario tested were identified. This emotional space consisted of two axes defined by the loading pattern of the individual physical reactions.

If, for instance, the participants' facial muscle activity had a particularly strong influence on their emotional state, this LEA parameter would determine one axis in the coordinate system. On the other hand, the second axis describes, for instance, the level of physical excitement, determined by the test subjects' heart rate variability and brain waves. Therefore, the arrangement of the lighting scenarios along the two axes ultimately also describes their specific emotional effect.

For each varying lighting parameter, separate discriminant analysis was carried out in order to explore the emotional effect of changes to individual lighting parameters 
(see Graphic 3 as a sample representation of a discriminant range for the "light colour" parameter).

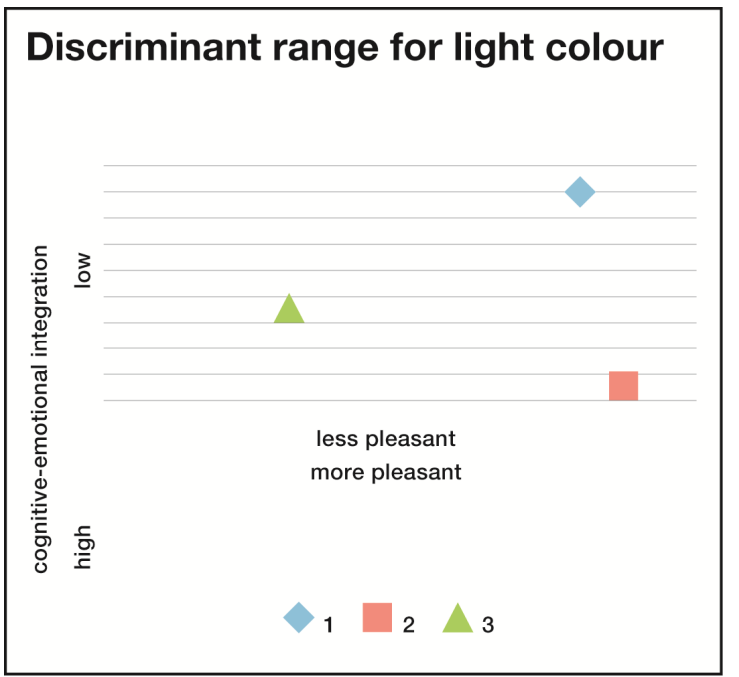

Graphic 3: Discriminant range for the "light colour" parameter. Localisation of lighting scenarios $1-3$

At the end of the analysis, each scenario was evaluated by parameter and target group, according to cognitive and emotional load in each case.

\section{Results}

As mentioned already, major differences were observed with respect to the lighting preferences of the individual target groups. For instance, it was clearly shown that there is no single lighting scenario that is preferred by all target groups in equal measure. While a least common denominator was defined across all target groups, there were nevertheless clear differences as to the target groups' individual lighting preferences.

These findings open up huge potential for developing lighting concepts customised to meet the needs of the target groups.

On a superordinate level, three groups each consisting of 2-3 Limbic $\AA$ Types with similar preferences as to the combination of lighting parameters were identified.

\section{a. Target group-specific results}

Group 1 - BALANCE: persons looking for harmony and relaxation (Harmonisers, Traditionalists, Open-minded)

The first identified group, "BALANCE", is dominated by the largest group among the Limbic $\AA$ Types, the Harmonisers; but this scenario was also concluded to be the most positive one for Traditionalists and Open-minded.

This rather quiet, harmony-focused family type showed particularly positive responses to moderate accent lighting. The lighting solution was dominated by a mixture of medium flood beam angles (20-309) at the POS and directly on the goods displayed. Within the solution, highly uniform vertical lighting on shelves, in peripheral zones and around displays, as well as integrated and dimmed accent lighting in coves and display cabinets proved to be a key factor of success. The ambient lighting was generated by reflection of the medium flood accent lighting. The proportion of diffuse light by downlights is very low. The horizontal general lighting level is $800 \mathrm{~lx}$ on average, the colour temperature is $3000 \mathrm{~K}$ for the most part.

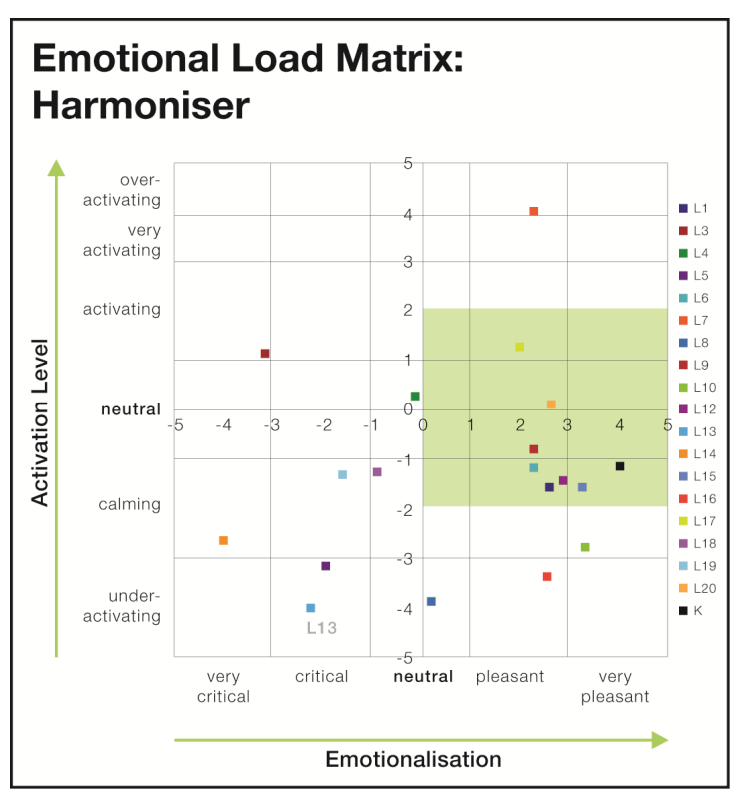

Graphic 4: Emotional load Harmonisers. The green zone marks the range of acceptance of this target group.

\section{Group 2 - STIMULANCE: unconventional persons} (Hedonists, Adventurers)

The second group comprises the fun-loving Hedonists and the risk-loving Adventurers. Especially Hedonists get bored quickly, they need variety and challenges in their lives. Lighting scenarios should facilitate a positive state of relaxation, but still keep a certain level of stimulance to avoid boring the target group. Here, for instance, scenarios offering high contrasts, with a reduced horizontal level of general lighting, will prove beneficial.

Specifically, it was found that these two Limbic $®$ Types show extremely positive responses to lighting scenarios with marked contrasts, generated through accent lighting and a combination of various different spots. Spotlights with beam patterns between $12^{\circ}-16^{\circ}$ (without Superspot $<10$ $\%)$ at the POS and directly on the goods displayed, as well as dimmed, uniform vertical lighting on shelves and displays were key in this respect. Moreover, to stimulant these two Limbic ${ }^{\circledR}$ Types, fully integrated accent lighting in coves, display cabinets and on shelves is important. The ambient lighting in circulation areas and the generally diffuse lighting level provided by downlights or coves are the lowest of the three groups in STIMULANCE. Accordingly, ambient lighting is only used for decorative purposes, for instance when cove lighting. The horizontal general lighting level is approximately $\sim 500 \mathrm{Ix}$. The amount of vertical light and the amount of light at the POS, on the other hand, are the highest among the three groups, thus providing for the required stimulation. The colour temperature clearly tends towards cool white, with $4000 \mathrm{~K}$ prevailing.

Group 3 - DOMINANCE: Critical persons (Performers, Disciplinarians)

The third group is characterised by the sceptics among the Limbic $\AA$ Types, in other words those that are easily unsatisfied and turn away from situations if they do not meet their expectations. This basically sceptical target group is sensitive to unbalanced lighting concepts and is best loaded with positive emotions through well-balanced, 
moderate lighting effects. For both types, accordingly, it is all the more important to achieve the emotional optimum, since adequate lighting is considered basic.

Both types showed the best responses to balanced light distribution, but were also susceptible to discreetly moodenhancing lighting effects. Over stimulation through narrowbeam lighting with extreme contrasts should be avoided.

These target groups showed particularly positive responses to lighting solutions featuring high uniformity as well as a combination of wideflood accent lighting with beam angles of $>35^{\circ}$ at the POS and directly on the goods displayed. Moreover, a high proportion of uniform vertical lighting on shelves and displays as well as integrated and dimmed accent lighting in coves and display cabinets were decisive to stimulate these subjects. The focus of accent lighting in this context is on uniform vertical lighting in peripheral areas. The general lighting level is made complete by downlights and backlit coves. The proportion of diffuse ambient lighting is the highest among the three groups. The colour temperature of the scenario with the highest stimulation potential ranges between warm white and intermediate, with a tendency towards $4000 \mathrm{~K}$.

\section{b. Summary of results and discussion}

The analysis clearly shows that each target group has its own optimum lighting scenarios. Overall, three major groups were identified, each with their own requirements as to the lighting solution: those looking for harmony and relaxation, the unconventional ones and the sceptical ones.

Designing the lighting scenarios along the lines of target group specific requirements is explicitly recommended in order to provide for the customer actually feeling at ease in the shop, perceiving positive emotions and being put into high-awareness states of mind.

Only if the customers find the atmosphere in a shop acceptable and even pleasant, they will also be motivated to stay a little longer. Ultimately, more time spent in the shop means that the customer is provided with more opportunities to notice the products and brands on display and to buy them.

The study has demonstrated that target groups respond to even the smallest changes in very different ways. While hardly noticeable with the naked eye, minor differences in the surroundings are perceived strongly by customers at an unconscious level, as the psychophysiological data have shown.

\section{Next steps}

Each brand, product and shop has it's specific target group. The analysis clearly shows that these target groups respond in different ways, and that they have quite individual needs, especially with respect to the lighting solution.

Accordingly, any lighting scenario needs to suit not only the brand or product advertised, but it also needs to match the target group. The unique findings of the present study allow for this next step to be taken towards improved and more targeted stimulation of different target groups. Moreover, it offers the opportunity to develop optimised lighting scenarios, luminaires and lighting technology with a view to a perfect brand and target group fit.

\author{
Authors: \\ Werner Bernd, Gruppe Nymphenburg Consult AG, ArtDeco Palais, \\ Arnulfstrasse 56, 80335 Munich, Germany, \\ email:info@numphenburg.de \\ Cornel Hess, Zumtobel Light GmbH, Schweizer Strasse 30, 6850 \\ Dornbirn, Austria, email: cornel.hess@zumtobelgroup.com \\ Pavel Šobra, ZG Lighting Czech Republic s.r.o., Jankovcova 2, \\ 17000 Praha 7, Czech Republic, \\ email:pavel.sobra@zumtobelgroup.com
}

